\title{
Erratum to: Non-metabolisable insulin glargine does not promote breast cancer growth in a mouse model of type 2 diabetes
}

\author{
Emily J. Gallagher ${ }^{1}$ - Zara Zelenko ${ }^{1}$ - Aviva Tobin-Hess ${ }^{1}$ - Ulrich Werner ${ }^{2}$. \\ Norbert Tennagels $^{2} \cdot$ Derek LeRoith $^{1}$
}

Published online: 23 February 2017

(C) Springer-Verlag Berlin Heidelberg 2017

\section{Erratum to: Diabetologia}

DOI 10.1007/s00125-016-4000-x

Unfortunately, there is an error in Fig. 1 and its accompanying legend. For the M1 metabolite, there should be no ' $R$ ' at position $\mathrm{B} 31$, and the third sentence of the legend has now been amended in line with this change. In addition, the final sentence of the legend has also been amended to explain the structures of insulin degludec and detemir more clearly.

The correct version of Fig. 1 and the legend are shown overleaf.

The online version of the original article can be found at http://dx.doi.org/ 10.1007/s00125-016-4000-x.

Emily J. Gallagher

emily.gallagher@mssm.edu

1 Division of Endocrinology, Diabetes and Bone Diseases, Department of Medicine, Icahn School of Medicine at Mount Sinai, One Gustave L Levy Place, Box 1055, New York, NY 10029, USA

2 Diabetes Research \& Translational Medicine, Insulin Biology, Sanofi-Aventis Deutschland GmbH, Frankfurt am Main, Germany 


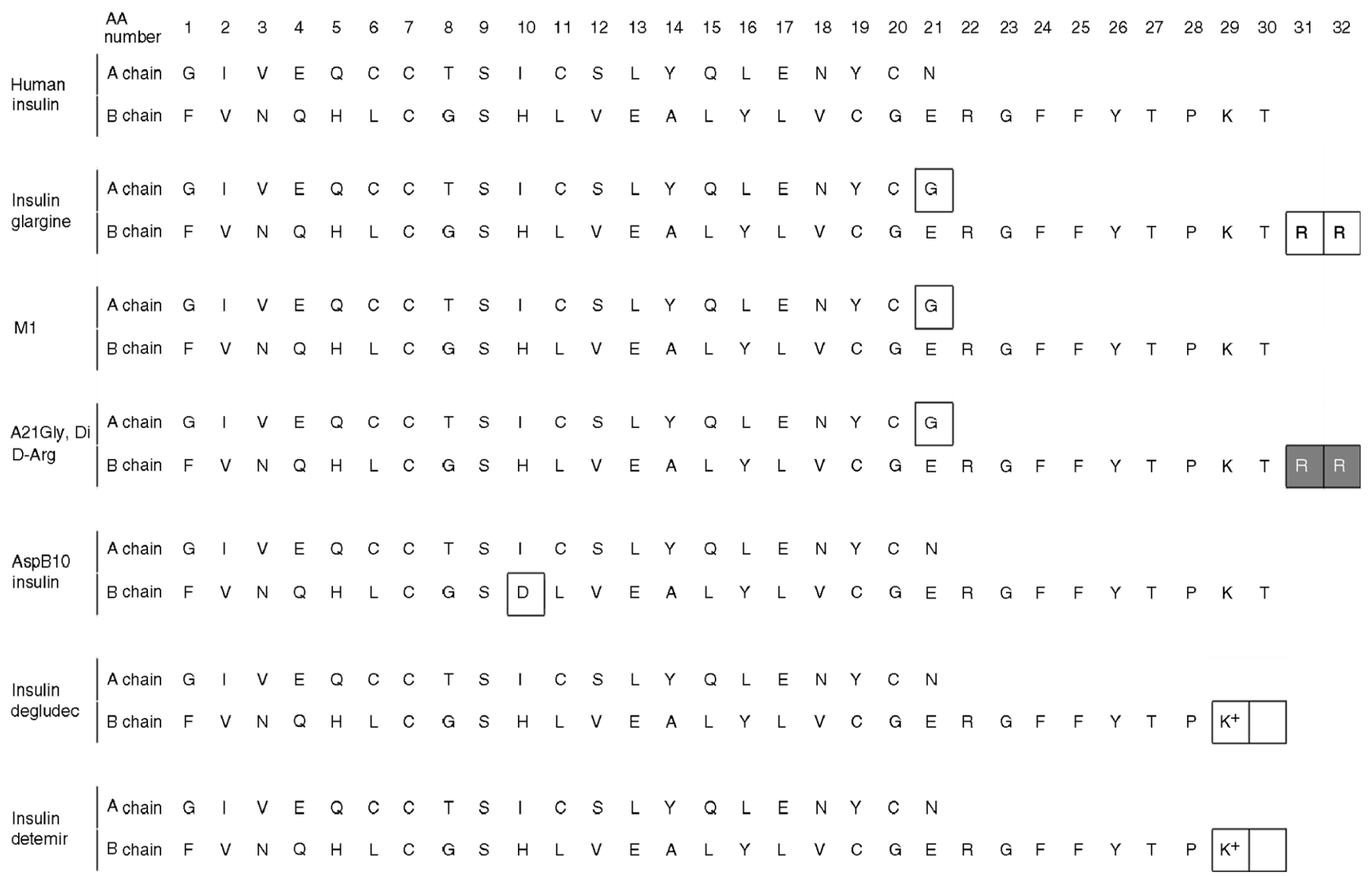

Fig. 1 Amino acid sequences of human insulin and insulin analogues. Human insulin comprises an A chain with 21 amino acids (AAs) and a B chain with 30 AAs. A box around the AA letter indicates that the AA is altered, removed or added in the analogue compared with human insulin. Insulin glargine is metabolised to the M1 metabolite by removal of the two C-terminal arginine residues. A21Gly,DiD-Arg has the same AA sequence as insulin glargine, but the $\mathrm{C}$-terminal arginines of parent insulin glargine are L-isomers (highlighted in bold font), and are D-isomers in non-metabolisable glargine (highlighted in grey). AspB10 insulin has an AA substitution of aspartic acid in place of histidine at position 10 on the $\mathrm{B}$ chain. $\mathrm{K}^{+}$at position 29 on the $\mathrm{B}$ chain of insulin degludec and insulin detemir indicates the linkage of a hexadecandioic acid side chain via a glutamic acid spacer (degludec), or a tetradecanoic acid (detemir) 